\title{
Politique
}

\section{La commission Macdonald : principes et préceptes}

\section{Raymond Hudon}

Numéro 9, hiver 1986

Démocratie et libéralisme

URI : https://id.erudit.org/iderudit/040517ar

DOI : https://doi.org/10.7202/040517ar

Aller au sommaire du numéro

Éditeur(s)

Société québécoise de science politique

ISSN

0711-608X (imprimé)

1918-6584 (numérique)

Découvrir la revue

Citer cet article

Hudon, R. (1986). La commission Macdonald : principes et préceptes. Politique, (9), 111-145. https://doi.org/10.7202/040517ar d'utilisation que vous pouvez consulter en ligne.

https://apropos.erudit.org/fr/usagers/politique-dutilisation/ 


\title{
La commission Macdonald: principes et préceptes
}

\author{
Raymond Hudon \\ Université Laval
}

Moins de trois ans après sa création, la Commission royale sur l'union économique et les perspectives de développement du Canada déposait son rapport en septembre 1985 . Il sera sans doute intéressant ultérieurement de voir quel sort aura été réservé à ses recommandations. Les réactions limitées enregistrées jusqu'ici laissent percer une certaine satisfaction dans quelques milieux économiques alors que la déception ou le désintéressement semblent plus largement répandus. Il faut dire que la création de la Commission ne paraissait pas répondre à un vœu vraiment populaire. Mais la participation du peuple canadien se révéla quand même importante. Le rapport déposé permet-il à ceux qui ont joué le jeu de s'y reconnaître ou d'y retracer les vues qu'ils avaient exprimées?

Après avoir montré que le Rapport Macdonald propose finalement un virage radical par rapport à des positions défendues à divers moments de l'histoire canadienne, la question globalement posée est de savoir si la route tracée pour parvenir à l'an 2,000 se révèle vraiment attrayante. Truffé de principes (de dogmes?), parfois teinté d'un certain "réalisme», le Rapport se présente finalement comme un recueil de préceptes d'enseignements plus que comme un programme de mobilisation en fonction d'objectifs 
palpables. Pour le montrer, quoi de mieux que de coller au contenu même du Rapport.

\section{Un virage: de Macdonald à Macdonald!}

Alors chef de l'Opposition, John A. Macdonald propose un amendement au discours du budget de 1878 pour énoncer un des volets importants de sa politique nationale présentée l'année suivante et formuler ses objectifs.

... the welfare of Canada requires the adoption of a National Policy, which, by a judicious readjustment of the Tariff, will benefit and foster the agricultural, the mining, the manufacturing and other interests of the Dominion; (...) such a policy will retain in Canada thousands of our fellow country-men now obliged to expatriate themselves in search of the employment denied them at home, will restore prosperity to our struggling industries, now so sadly depressed, will prevent Canada from being made a sacrifice market, will encourage and develop an active interprovincial trade, and moving (as it ought to do) in the direction of a reciprocity of tariffs with our neighbours, so far as the varied interests of Canada may demand, will greatly tend to procure for this country, eventually, a reciprocity of trade. ${ }^{1}$

Un an plus tard, devenu premier ministre, Macdonald articule cette politique tarifaire protectionniste à d'autres éléments pour ainsi mettre en place la «National Policy» qui marque plusieurs décisions politiques et économiques durant les décennies suivantes. Il faut bien sûr rappeler que les libéraux se font élire en 1896 avec l'intention d'accélérer le retour à la réciprocité qui avait prévalu entre 1854 et 1866. Mais les Américains ne semblent pas disposés à réviser la position prise en 1866 de rappeler unilatéralement le Traité de réciprocité. Quand en 1911, le Congrès américain montre un changement d'attitude destiné à redéfinir le

1. Extrait reproduit dans The Financial Post, 9 mars 1985, p. 46. 
cadre des relations commerciales entre le Canada et les ÉtatsUnis, ce sont les voteurs canadiens qui appuient la résistance manifestée par les Conservateurs appelés à remplacer le gouvernement libéral sortant.

À part quelques réductions plutôt ponctuelles et sectorielles, la politique tarifaire de 1879 contribue donc fortement à l'orientation des relations économiques entre le Canada et les États-Unis tout en ne bloquant pas le processus d'intégration de l'économie canadienne à l'économie continentale. Le développement industriel continue sa progression au Canada selon les attentes de John A. Macdonald, mais il se trouve pour une part toujours croissante par la pénétration du capital américain. Le commerce extérieur du pays tend à se concentrer de façon plus poussée du côté des États-Unis malgré les barrières tarifaires qui voient tout de même leur importance sensiblement réduite par l'accentuation de la libéralisation du commerce international au moyen des négociations multilatérales. La tendance à l'intégration continentale s'accélère dans l'aprèsguerre, tant et si bien que l'inquiétude se dessine face à la réalité de plus en plus oppressante d'un rapport de dépendance traduisant la position du Canada vis-à-vis son voisin du sud.

Cette inquiétude trouve ses premiers échos dans la deuxième moitié des années cinquante à l'occasion des audiences de la Commission royale d'enquête sur les perspectives de développement économique du Canada. L'accueil mitigé réservé aux mesures introduites par celui qui avait présidé les travaux de cette commission, Walter Gordon, montre la force d'attraction des États-Unis dont le leadership mondial est explicitement reconnu en 1965 à travers le Rapport Merchant-Heeney intitulé Principes de collaboration entre le Canada et les États-Unis. Les observations et conclusions du Groupe d'études ad hoc sur la structure de l'industrie canadienne (Rapport Watkins) font par ailleurs ressortir clairement en 1968 les effets limitatifs de certains aspects des rapports étroits entretenus 
entre le Canada et les États-Unis, spécialement en ce qui touche l'élaboration de politiques économiques nationales.

Quand Pierre Elliott Trudeau accède au poste de premier ministre en 1968, il commande une étude en profondeur de la politique étrangère canadienne. Un Livre blanc, Politique étrangère au service des Canadiens, constitue en 1970 l'aboutissement de cette révision. La question de l'indépendance politique du Canada est posée; il semble convenu de ne plus la soumettre exclusivement aux seuls impératifs économiques.

En tissant la trame complexe et vitale de leurs relations avec les ÉtatsUnis, les Canadiens doivent procéder à des choix minutieux, s'ils veulent résoudre à leur satisfaction les conflits qui surgissent entre leur désir de maintenir un haut niveau de vie et celui de garder leur indépendance politique $^{2}$.

Dans le Rapport Wahn, préparé aussi en 1970, l'indépendance n'est plus réservée au seul champ proprement politique.

... non content de garder son indépendance politique, le Canada doit aussi maintenir un degré suffisant d'indépendance sur le plan militaire, économique et culturel afin qu'il puisse dans la pratique, prendre les initiatives personnelles propres à tout pays indépendant qui se respecte ${ }^{3}$.

Affirmant nettement sa préférence pour «des politiques dynamiques, aux vues larges» plutôt que pour des mesures restrictives, le Comité Wahn invite toutefois à la prudence.

... le Comité reconnaît que, pour le moment, une attitude mixte s'impose. Afin de donner le temps aux politiques positives de produire leurs effets et peut-être aussi rassurer les Canadiens en leur montrant que le Canada

2. Gouvernement du Canada, Politique étrangère au service des Canadiens, Information Canada, Ottawa, 1970, p. 25.

3. Comité permanent des affaires extérieures et de la défense nationale de la Chambre des communes, Comité spécial au sujet des relations canado-américaines, Rapport à la Chambre, (Rapport Wahn), Deuxième session de la vingt-huitième législature, 19691970, Fascicule no. 33, Imprimeur de la Reine, Ottawa, 1970, p. 23. 
a l'œil sur les dangers d'une trop grande dépendance à l'égard des ÉtatsUnis, le Comité croit à la nécessité d'adopter une certaine attitude défensive dans nos relations avec les États-Unis ${ }^{4}$.

Cette attitude défensive guide nettement les mesures que les États-Unis décident d'adopter en août 1971 pour le rétablissement de relations plus avantageuses avec le reste du monde, le Canada y compris. Des choix s'imposent; en s'occupant de leurs affaires, les Américains contraignent les Canadiens de voir aux leurs d'encore plus près. De trois options identifiées disponibles, deux sont formellement rejetées: le statu quo et «une intégration plus grande avec les États-Unis». Est plutôt privilégiée une troisième option qui «a pour objectif fondamental de réduire, à la longue, la vulnérabilité de l'économie canadienne à l'égard des facteurs externes, et en particulier l'influence des États-Unis et, par là, de renforcer notre capacité de promouvoir les grands objectifs canadiens et d'assurer le sentiment de notre identité nationale»" 5 . Provoqué par un climat de quasi-panique et alimenté par la conviction d'une intervention nécessaire d'urgence, le choix de la troisième option en peut relever du seul coup de tête et doit s'appuyer sur une volonté d'action à long terme dans une perspective élargie.

Il n'existe aucune possibilité que l'on surmonte du jour au lendemain la lourde dépendance du Canada envers les États-Unis en matière de commerce, d'investissements et de technologie. Mais il n'y a aucune raison de ne pas entreprendre, dans le cadre d'une économie et d'un commerce en expansion, une réorientation partielle qui, pourrait à la longue diminuer la dépendance du Canada à l'égard d'un seul marché et, partant, rendre moins vulnérable l'économie canadienne elle-même. Un Canada plus fort, résultat d'une telle politique, voilà l'objectif que vise la troisième option. Il ne fait aucun doute toutefois que pour atteindre à cette diversification, même à l'échelle modeste que l'on propose ici, il faudra conjuguer la

4. Idem.

5. Mitchell Sharp, "Relations canado-américaines: Choix pour l'avenir», dans Perspectives internationales, numéro spécial, automne 1972, p. 20. 
politique commerciale à d'autres politiques - comme en matière de croissance industrielle et de propriété étrangère - qui s'attaquent aux problèmes particuliers que pose la situation nord-américaine ${ }^{6}$.

Plusieurs s'interrogent sur les effets réels de cette orientation politique en soutenant que les moyens mis en œuvre pour la traduire se révèlent insuffisants. Un long débat pourrait prendre place autour de cette évaluation. Il convient cependant de noter que des pressions se développent en faveur de l'introduction d'un accord de libre-échange entre le Canada et les États-Unis: les arguments se greffant à cette option sont repris et articulés dans un rapport préparé par le Conseil économique du Canada en 1975 (Au-delà des frontières) et dans deux rapports en 1978 et 1982 du Comité sénatorial permanent des affaires étrangères, présidé par le sénateur George C. van Roggen. Dans un document d'orientation sur la politique commerciale, le gouvernement exprime cependant en 1983 ses réserves sur une telle option, se montrant quand même disposé à envisager la négociation d'un accord de libreéchange sectoriel avec les États-Unis et d'autres pays.

L'option de libre-échange a été une question litigieuse tout au long de l'histoire du Canada, moins pour des considérations économiques que pour des questions de souveraineté et d'auto-détermination. Les témoignages recueillis jusqu'à maintenant sur la nécessité d'y donner suite ne sont pas convaincants et un appel au libre-échange n'obtient pas un large appui. La plupart des analyses tendent à insister sur les avantages économiques pour le Canada et ne tiennent pas pleinement compte des coûts ou conséquences, politiques et économiques. Toutefois, l'option demeure et pourrait ultérieurement obtenir des appuis plus nombreux lorsque des circonstances différentes engendreront des attitudes différentes. Il est néanmoins possible de satisfaire à bon nombre des arguments en faveur du libre-échange en concluant progressivement des accords bilatéraux visant à résoudre des problèmes particuliers comme ceux qui ont résulté de l'imposition par les États-Unis de restrictions concernant la vente de matériel canadien de transport en commun et de tarifs s'appliquant aux exportations canadiennes de produits pétrochimiques. Le libre-échange

6. Ibid., p. 26. 
avec les États-Unis sur une base sectorielle limitée ne poserait pas les problèmes plus complexes qui découleraient de l'option du libre-échange global et serait conforme à l'orientation progressive des gouvernements canadiens qui se sont succédés vers la libéralisation du commerce. Cette façon de procéder ne doit pas nécessairement se limiter au Canada et aux États-Unis, mais peut s'impliquer à d'autres pays intéressés?

Ce rappel sommaire de positions successives prises par le gouvernement canadien et d'autres instances gravitant autour des centres de décision sert bien à placer en perspective les conclusions du rapport déposé à l'automne 1985 par la Commission Macdonald.

Les commissaires pensent que des négociations avec les États-Unis ne constituent en soi ni une panacée ni une menace mais plutôt une solution de prudence qui contribuera à nous enrichir et, par là même, à renforcer la structure de notre pays et à augmenter notre confiance en nous-mêmes. Même si, initialement, ce choix accroissait la dépendance du Canada envers le marché américain, il offrira à notre pays une relation plus sûre et nous rendra encore moins vulnérables. En définitive, cette option devrait fortifier et diversifier notre économie en nous permettant de réaliser des objectifs que nous poursuivons depuis longtemps mais qui nous ont toujours échappé, principalement parce que notre propre secteur manufacturier a toujours été trop faible pour que nous puissions les atteindre ${ }^{8}$.

Pour dire le moins, le Rapport Macdonald invite le peuple canadien à effectuer un virage prononcé touchant les perspectives de développement du Canada. Si John A. Macdonald voyait dans l'établissement de barrières tarifaires le moyen de favoriser le développement industriel au Canada, la Commission présidée par Donald S. Macdonald soutient que c'est plutôt leur abolition qui

7. Gouvernement du Canada (Affaires extérieures). La politique commerciale du Canada pour les années 80, Document de travail, Approvisionnements et Services Canada, Ottawa, 1983, p. 50.

8. Commission royale sur l'union économique et les perspectives de développement du Canada, Rapport, (Rapport Macdonald), Approvisionnements et Services Canada, Ottawa, 1985, Volume I, p. 418. Pour ne pas alourdir démesurément l'appareil de références, tout renvoi ultérieur au Rapport Macdonald sera indiqué dans le texte même par l'identification du volume (I, II ou III) et de la page ou des pages d'où est tiré l'information ou l'extrait cité. 
permettra l'atteinte du même objectif. À un peu plus de cent ans d'intervalle, on peut saisir que le détour proposé par John A. Macdonald pour aboutir à un accord de réciprocité est maintenant perçu comme inapproprié. Les programmes de construction d'économies nationales s'estompent devant la tendance à la mondialisation de l'économie et cela dans un contexte de libéralisation toujours plus accentuée. La prudence qui, il y a seulement un peu plus de dix ans, incitant à privilégier certaines options contre d'autres semble plutôt indiquer, actuellement, leur rejet pour réintroduire celles antérieurement considérées comme téméraires. Les rapports de dépendance auparavant jugés comme de sérieuses limites semblent devoir constituer désormais la source de nouvelles possibilités et de forces. Les «théoriciens» de la dépendance avaient pourtant été prévenus que leurs schémas explicatifs causaient problème. Enfin, fini le temps des compromis inspirés de considérations politiques et culturelles; les solutions économiques ne représententelles pas des solutions universelles? C'est maintenant bien affirmé: être plus dépendant, c'est être moins vulnérable!

Le rapport Macdonald: La route vers l'an 2000?

Une commission, un mandat, un rapport

Il ne faut pas se méprendre; le Rapport Macdonald ne traite pas de la seule question des relations canado-américaines. Les réactions qui ont suivi son dépôt ont tout de même été fortement concentrées autour de cet enjeu et de l'option y étant rattachée, le libre-échange. Il demeure surprenant que ces réactions n'aient pas été plus nombreuses et surtout plus diversifiées. Il est vrai d'une part que les interventions du gouvernement Mulroney ont largement contribué à attirer l'attention sur un projet de libreéchange avec les États-Unis. D'autre part, le déroulement d'une campagne électorale au Québec, malgré une intensité moindre 
que celle habituellement observée dans la période récente, ne constitua pas une occasion propice au débat autour des orientations proposées par la Commission.

Faut-il applaudir ou déplorer? Il faut au moins signaler que la somme d'énergies mobilisées par la réalisation du mandat de la Commission aurait justifié des réactions plus nombreuses et des discussions publiques plus importantes. "Tous les mémoires et les transcriptions des audiences publiques [...] assemblés en 96 volumes» $(\mathrm{I}, \mathrm{xx})$ laissaient voir une participation importante de la population canadienne aux travaux de la Commission. Il est bien possible que cette population ne soit pas retrouvée à travers les nombreuses pages (près de 2,000) rendant compte de ce que Donald Macdonald désigne comme «la consultation et la recherche la plus importante jamais réalisée au Canada» (III, 649) dans une lettre qu'il adressait le 7 septembre 1984 au nouveau premier ministre élu, Brian Mulroney.

Gérald Docquier, en faisant état de ses divergeances touchant un certain nombre des conclusions contenues dans le Rapport, estime que la recherche, alimentée de la «nouvelle orthodoxie» de l'autosuffisance et des forces du marché, a nettement primé sur la consultation: "Tandis que les commissaires se préoccupaient des expériences quotidiennes des Canadiens, le personnel de la Commission amorçait un programme de recherche, qui était sensiblement détaché des réalités exprimées par ceux qui se présentaient devant nous». (III, 602-603). En rassemblant quelques-uns des mémoires présentés à la Commission et véhicules de certains consensus sur l'avenir du Canada9, Daniel Drache et Duncan Cameron ont cherché à montrer la justesse des propos de Docquier

9. Daniel Drache et Duncan Cameron (eds), The Other Macdonald Report. The Consensus on Canada's Future that the Maidonald Report Left Out, James Lorimer \& Company, Toronto, 1985. 
qui juge, en dernière analyse, que «la Commission n'est pas parvenu [sic] à réaliser son mandat» (III, 612).

Une lecture du texte de l'arrêté-en-conseil du 5 novembre 1982 (III, 639-643) fait voir l'ampleur et la complexité du mandat confié à la Commission. Il avait été demandé aux commissaires de garder à l'esprit quelques principes devant guider leur travail d'enquête et de rédaction, dont celui de tenir compte des «aspirations plus générales» des Canadiens. Les commissaires confessent que leurs efforts ne parvinrent pas à découvrir les lois universelles qui régissent les comportements économiques et politiques.

Nous n'avons pas découvert un message unique et non équivoque, simple s'imposant de lui-même. Les modèles que nous avons trouvés, et auxquels nous avons dû réagir, sont d'une complexité décourageante. Les Canadiens poursuivent des objectifs multiples, tant sur le plan national que sur la scène internationale. Nous avons donc dû formuler une foule de réponses aux nombreuses questions relevant de notre mandat. (I, 5-6).

Les réponses formulées laissent voir des influences nettes, mais pas toujours concordantes. Leur intégration, leur agencement apparaissent toutefois inachevés. En réalité, l'impression se dégage que les commissaires se sont trouvés dans une situation similaire à celle qu'ils observent dans l'ensemble de l'activité gouvernementale: «Le pouvoir se déplace vers les spécialistes et ceux qui contrôlent l'information». (I, 39). Ainsi, selon les sujets et problèmes abordés, il est possible de repérer la trace des économistes, juristes et politicologues, le premier groupe marquant plus profondément les orientations du Rapport.

Il est régulièrement plus facile de convaincre ou de passer un message quand l'impression est crée qu'est ainsi traduite une certaine unanimité, une certaine homogénéité idéologique certains diraient peut-être unité théorique et méthodologique. Un sondage réalisé par The Financial Post ${ }^{10}$ auprès des économistes

10. Voir "What the economists say about free trade with U.S.", dans The Financial Post, 26 octobre 1985, p. 1. 
actifs dans le milieu des affaires au Canada ne montrait-il pas que 84 pourcent d'entre eux se déclaraient favorables à un accord de libre-échange avec les États-Unis? Dans une proportion de trois sur quatre, ces mêmes économistes croyaient de plus qu'un tel accord de libre-échange avait peu de chance de conduire à l'intégration politique.

Même en supposant que les opinions diversifiées exprimées par la population canadienne se trouvent mal représentée au sein du Rapport déposé, il se révèle inexact de suggérer que les commissaires oublièrent cette même population et ses représentants dans la réalisation de leur mandat. Renonçant à exercer la fonction d'écho fidèle à cause de la "complexité décourageante» de la tâche, les commissaires choisirent finalement de faire œuvre d'éducation conformément à ce que les politicologues enseignent généralement sur le rôle des commissions d'enquête.

Le rôle d'une Commission royale consiste donc surtout à faire œuvre d'éducation. Elle prend du recul par rapport au processus politique, présente une perspective différente et suggère des principes généraux qui pourront guider ceux qui auront à faire les choix politiques de l'avenir. (I, xxii)

Il semble tout à fait pertinent de cerner plus précisément la perspective et les principes proposés par la Commission. Car si l'objectif d'éducation devait réussir, la projection du Canada de l'an 2000 pourrait alors être esquissée.

\section{La domestication du politique}

La perspective adoptée par la Commission Macdonald differe au moins sur une des traits importants de l'histoire politique canadienne. Il n'est plus vraiment question de faire exercer ni même de laisser l'État exercer un rôle déterminant dans l'élaboration de la politique économique. 
... les commissaires estiment que les Canadiens doivent, dans certains domaines importants, s'en remettre bien davantage aux lois du marché. Nos propositions de nous ouvrir davantage à l'économie internationale et, plus particulièrement, de conclure un accord de libre-échange avec les États-Unis, traduisent notre préférence pour les lois du marché, par opposition à l'intervention de l'État, comme moyen de stimuler l'économie et la croissance. (I, 72).

Le message est clair. Selon les termes utilisés par les commissaires, "le temps où les gouvernements pouvaient croître et se réserver une part plus importante d'un gâteau de plus en plus grand est révolu». (I, 77). Il faut par ailleurs suivre le raisonnement. Parce que le monde change, il faut «des changements réels pour venir à bout des problèmes actuels" (I, 218). Les commissaires semblent d'autant plus horrifiés par la perspetive d'un refus du changement, d'une volonté de maintien du statu quo, qu'une telle orientation politique "pourrait nous conduire très rapidement, au cours des quelques prochaines années, à réclamer l'institution d'une économie planifiée» (I, 299). On entre alors dans une sorte de cercle vicieux: ne pas changer exigera plus d'interventionnisme, et le changement deviendra alors de plus en plus improbable. Car les gouvernements seraient «naturellement» conservateurs.

Un système politique puise à un certain nombre de valeurs et d'idées et il doit évoluer avec elles. La démocratie ne se contente pas de subir ce genre d'évolution; elle la cultive. Cette stimulation de l'expérimentation et de l'innovation ne s'exerce pourtant qu'à la marge. Elle est contrée par l'inertie massive des gouvernements tentaculaires. Les idées et les valeurs d'hier sont déjà bureaucratisées et inscrites dans les programmes qui résistent au changement. Presque tout ce que font les gouvernements représente la continuation de programmes antérieurs. Les nouveaux programmes ne constituent jamais qu'une petite proportion de l'activité totale du gouvernement. Ainsi, les gouvernements en place sont inévitablement des instruments de conservatisme, plus enclins à l'habitude qu'à l'innovation. (I, 29).

Les commissaires souhaitent en fin de compte que gouverner, ce soit véritablement prévoir, accepter et assimiler le changemnt. 
Leur vision de la pratique indique que c'est l'inverse qui se produit: on s'accroche au passé, on résiste au changement. S'il y a une porte de sortie, elle se trouve du côté du marché; c'est là que l'évolution se réalise. C'est de ce niveau que se dégage la perspective d'ensemble essentielle à l'élaboration de plans d'avenir, de programmes de changement. Selon les commissaires, «le fonctionnement quotidien du gouvernement [...] s'écarte inévitablement d'une perspective d'ensemble. Les décisions des gouvernements sont généralement assujetties aux pressions du moment, et cellesci ne facilitent pas l'adoption d'une perspective ouverte sur l'avenir». $(I, 6)$ Comment alors interpréter l'évolution des positions des commissaires entre le début et la fin de leur mandat? Donald Macdonald soutient que le changemnt de gouvernement en septembre 1984 compte beaucoup moins que l'«évolution personnelle» des membres de la Commission. (Après tout, les changements de gouvernement ne sont pas aussi significatifs qu'on le croit.) On a cependant fait remarquer que "personne à la Commission Macdonald ne nie que l'impulsion donnée par le nouveau gouvernement conservateur à certains dossiers a eu un impact sur la réflexion des 12 commissaires " ${ }^{11}$. Donald Macdonald suggère en quelque sorte qu'il n'y a pas de mal à ajuster ses options à une situation changeante ${ }^{12}$. Le Rapport Macdonald demande bien simplement aux politiciens d'en faire autant.

Mais on vit dans un régime démocratique dont la vitalité dépend en principe "de la nature de la relation qui s'établit entre cabinets et parlements ou législatures, d'une part, et, d'autre part, entre citoyens et parlements ou législatures» (I, 75). Cela risque toutefois d'être affaire de quotidienneté, car l'État agit

11. Paule Des Rivières, "Une fin de mandat marquée par un changement de cap radical. Les positions de la Commission Macdonald reflètent celles du gouvernement conservateur", dans Le Devoir, 23 février 1985, pp. 1, 10.

12. Voir "Macdonald: The man and his report", dans The Financial Post, 14 septembre 1985 , p. M3. 
aussi «comme arbitre sur la scène sociale où divers groupes luttent constamment pour promouvoir leurs intérêts [en utilisant] l'État pour faire avancer leur cause» $(I, 12)$. Et le conflit n'est pas en soi répréhensible. Tout au contraire...

Dans une société pluraliste ouverte, où les politiques nationales conferent délibérément ou involontairement avantages et désavantages, les conflits et la rivalité entre les divers intervenants privés désireux d'influencer les gouvernements sont inévitables et fonctionnels. Ceux-ci fournissent aux gouvernements les informations dont ils ont besoin pour élaborer de nouvelles politiques et modifier les anciennes. (III, 9)

Si les commissaires admettent que "le conflit est propre à notre système et reflète des différences réelles», ils refusent par ailleurs d'en faire l'éloge parce qu'il ne comporte pas «que des aspects positifs»: «La jungle darwinienne n'est pas notre idéal, ni celui du Canada». (idem)

Voilà pour la "politique ordinaire» (III, 19). Il y a aussi la politique véritable, celle qui tient compte des nouvelles réalités économiques internationales. Et c'est là que l'œuvre d'éducation intervient, pour dissiper tout doute quant au volet qui doit s'imposer.

La relation entre l'État et le marché, entre la société politique et l'économie, comporte une dimension internationale aussi bien que nationale. Pour un pays qui dépend autant du commerce international que le Canada, cette dimension revêt une importance particulière. Aujourd'hui comme par le passé, les changements intervenus à l'échelle de l'économie globale exigent des Canadiens qu'ils réagissent positivement à un nouveau contexte international. La réponse qui s'impose exigera une profonde adaptation de notre économie pour faire face à la concurrence internationale; un réajustement de nos politiques sociales et industrielles pour soutenir cette adaptation; une négociation bilatérale vigoureuse pour obtenir un accès plus sûr au marché américain, alliée à des démarches multilatérales permanentes; des modifications constitutionnelles du fédéralisme canadien en vue d'accroître notre efficacité dans la négociation et la mise en œuvre d'ententes commerciales; l'adoption de contre-mesures en vue de préserver effectivement notre autonomie politique, à mesure que nous nous ouvrons davantage aux forces concurrentielles de l'étranger. $(I, 63)$ 
En somme, pour parer au "caractère quelque peu improvisé des politiques gouvernementales» (I, 61), il faut domestiquer l'État, le soumettre à des contraintes qui l'amèneraient à dépasser les pressions du moment. Ce qui ne signifie pas que l'État se révèle inutile. Il a, entre autres, "le devoir de promouvoir l'intégration sociale» (III, 17) non pas en cherchant "à réduire ou à éliminer les activités [des] groupes d'influence», mais en poussant le "processus de réconciliation» (III, 45). Compte tenu des orientations privilégiées par la Commission quant au rôle de l'État, il peut être embêtant de constater que les «mécanismes de consensus» aient particulièrement réussi dans des pays gouvernés par des sociaux-démocrates (II, 378). Mais ni le tripartisme, ni la corporatisme ne sont conçus comme des solutions acceptables; ils peuvent plutôt présenter de sérieux dangers (III, 60) et encourager des "politiques élitistes» (III, 62). C'est le cas aussi d'une consultation trop poussée qui gênerait «le processus d'adaptation qui favorise le développement économique» (III, 61).

L'État n'est pas appelé à disparaître; il doit être simplement adapté, son rôle doit être redéfini conformément aux exigences économiques plutôt qu'être limité par des jeux politiques quasi inévitablement à courte vue. La gestion publique doit s'inspirer du modèle de la gestion privée; l'État doit remplir une fonction essentielle de direction plutôt que de sombrer dans le dirigisme. Dans ces conditions, le premier ministre pourra agir au même titre qu'un "président directeur général» dans une entreprise (III, 48). De même, on pourra prévenir les plus graves distorsions dans le fonctionnement des lois du marché.

\section{La préservation de l'État - appui}

Une fois que l'on s'est bien convaincu que trop de politiques sont à la source «des rigidités de nos marchés du travail, [d'] une protection généralisée des secteurs péréclitants de notre économie 
qui va bien au-delà des mesures transitoires appropriées, de même que des systèmes de commercialisation qui haussent les prix et les coûts» (I, 72), il convient de faire ressortir que l'État n'est pas que nuisance. Il est un produit historique, "le rôle qui lui est dévolu de nos jours au Canada est le résultat cumulatif involontaire de milliers de décisions et de politiques particulières qui se sont échelonnées sur un demi-siècle» $(I, 71)$.

Un discours absolutiste réclamerait le retrait de l'État de toute activité économique. Par contre, «on peut justifier une intervention gouvernementale dans des situations où existe ce que les économistes appellent souvent une défaillance du marché» (II, 283). Le marché ne constitue donc pas la solution parfaite, même si on veut poser que le libre jeu des forces du marché représente la situation idéale. Même que pour s'exercer de façon optimale, le libre jeu de ces forces requiert une présence active de l'État.

Les marchés ont également besoin des États, car ils ne peuvent se développer que s'il y a ordre, respect de la loi, respect du droit de propriété, application des contrats, fiabilité des poids et des mesures, stabilité de la monnaie, infrastructure pour le transport et autres services que les marchés ne fournissent qu'avec difficulté. (I, 46).

Malgré le principe de la primauté du marché, l'État a donc sa place en amont de ce marché en vue d'assurer les conditions requises pour son bon fonctionnement aussi bien qu'en aval pour corriger ses défaillances les plus graves. La marge de manœuvre des gouvernements est en quelque sorte définie par la tâche qui leur incombe de «trouver les options qui réduisent le conflit existant entre les politiques qui sont dictées par de bonnes considérations politiques et celles dictées par de bonnes théories économiques et de bonnes préoccupations sociales» (II, 271). Encore une fois, il faut constater que les rapports entre l'économique et les autres volets de l'activité humaine ne peuvent être que conditionnés par le statut théorique conféré à la sphère économique. 
En adhérant aux bonnes théories économiques, l'État pourra se lancer dans des opérations de consultation qui, sans se traduire «nécessairement par des accords», pourront «influencer les décisions prises par les groupes participants»; car «la consultation, c'est aussi la formation mutuelle». (III, 59).

Dans nombre de situations, les gouvernements ont l'obligation de «réduire la résistance au processus de changement lui-même» par exemple, «en aidant les travailleurs à s'ajuster aux changements » (II, 268). À la condition expresse qu'elle ne constitue pas «une entrave à l'adaptation au marché du travail [en encourageant] les prestataires à demeurer dans des régions ou des professions où les perspectives d'emploi sont mauvaises» (II, 663-664), il est ainsi admis que l'assurance-chômage doive «être fournie par l'État» (II, 665). Adopter des mesures visant à facilité l'adaptation du travail au changement ou simplement réduire sa résistance ne doit pas néanmoins entraîner que l'on se substitue aux mécanismes de négociation au sein des entreprises: "Les restrictions législatives traditionnelles sur le recours au droit de grève ou au lock-out ont fait leur temps»(II, 774). Même dans les cas extrêmes de mésentente, l'intervention la moins drastique doit encore être privilégiée: «La Commission est d'avis que le recours répété à des législations de retour au travail est probablement plus nuisible à la négociation que le recours à l'arbitrage»(II, 777). Quand l'intervention se situe à un niveau général d'assistance au marché, il peut être exigé «que le gouvernement en prenne l'initiative» comme dans le cas des changements à apporter aux mécanismes de détermination des salaires (II, 398) ou il peut exister «de bonnes raisons [pour, par exemple,] mettre en œuvre des politiques visant à encourager les ententes de participation aux bénéfices » (II, 410). Les effets de stabilisation découlant de telles interventions rendent tolérables les entorses aux principes du noninterventionnisme. 
En plus d'assurer les conditions propices à l'exercice des lois du marché, l'État est encore invité à intervenir pour corriger les effets du libre jeu des forces du marché. Ainsi, les commissaires se déclarent "parfaitement conscients du nombre d'exemples d'échecs sociaux et économiques liés aux lois du marché, de situations où le marché du travail ne permet pas d'utiliser efficacement les ressources humaines» (II, 595). Relater les positions de la Commission sur l'État-providence, oblige à réaliser qu'il serait relativement inexact de simplement assimiler ses orientations au néo-conservatisme dogmatique.

L'État-providence est nécessaire pour résoudre les problèmes et les conflits sociaux qui sont inhérents à toute grande société industrialisée. Ces problèmes ne peuvent être résolus par la philantropie. Sans État-providence, ni la stabilité sociale, ni l'intégration sociale ne seraient possibles. Sans la contribution - stabilisante et propice à l'intégration - de l'Étatprovidence, le soutien à l'autonomie relative des marchés capitalistes s'écroulerait (II, 50).

À toute fin pratique, quant l'État intervient en aval pour corriger certaines défaillances du marché, son intervention sert aussi en amont en améliorant les conditions de fonctionnement de ce même marché. La position de la Commission se défend assez aisément à partir du moment où est retenu que «l'expansion de l'État-providence canadien - qui semble spectaculaire quand on l'examine isolément - paraît beaucoup plus modeste quand on la compare à la norme générale des nations industrialisées occidentales» (II, 612). Les idéologues de la droite, qui ont peu tendance à s'appuyer sur les faits ${ }^{13}$, se trouvent ainsi invités à reviser leurs «dogmes».

Généralement, les commissaires s'entendent pour dire qu'il n'y a aucune raison de croire que l'État-providence canadien ne puisse subsister ou qu'il impose une charge insurmontable à notre économie. La comparaison

13. À ce sujet, voir Robert Kuttner, "The Poverty of Economics", dans The Atlantic Monthly, février 1985, pp. 74-84. 
avec d'autres pays démontre que s'il y a une limite évidente quant aux dépenses permises dans une économie de marché, le Canada ne l'a pas encore atteinte. D'autres nations occidentales ont réussi à allier des dépenses sociales plus élevées que les nôtres, en regard du PNB, à une performance économique impressionnante (II, 636).

Compte tenu du bénéfice non négligeable que peut fournir au marché la prestation de services sociaux, on peut comprendre que les commissaires expriment «quelques réserves concernant la mise en œuvre trop zélée» d'une formule de privatisation de ces services (II, 891). En dépit d'une préférence globale de principe pour l'activité privée par rapport à l'activité publique, le secteur des services sociaux n'est pas le seul touché par une attitude plus pragmatique. Pour illustrer, on signale les problèmes que pourrait engendrer «la vente au secteur privé des forêts appartenant à la Couronne» pour finalement inviter à «concevoir des systèmes de propriété de la terre autres que la propriété privée» et à tenir compte de la nécessité «d'augmenter dans des proportions importantes les dépenses publiques destinées au renouvellement de la forêt, à sa protection et à sa gestion» (II, 493). Une position comparable est formulée en ce qui touche la préservation de l'environnement, matière pour laquelle «les commissaires estiment qu' [...] une plus grande intervention s'avérera nécessaire» (II, 582).

Enfin, il apparaît impossible d'ignorer certaines réalités politiques qui échappent au contrôle de la théorie et de l'idéologie. Ainsi, touchant le problème des inégalités régionales au pays, on peut être amené à suggérer, contre tout principe, que «le gouvernement fédéral pourrait fournir des emplois ou des investissements dans les régions déprimées, par exemple, en subventionnant les entreprises privées» (II, 253). Par ailleurs, on peut se retrouver forcé de répondre à certaines formes d'interventions par des interventions dont pourtant le recours répugne. Si d'autres pays subventionnent leurs propres industries, il est affirmé préférable de tenter d'obtenir l'abandon de telles pratiques. En cas d'échec, 
cependant, il convient d'envisager l'attribution de contre-subventions pouvant «inciter «l'autre partie» à abandonner sa politique interventionniste» (II, 284).

Les commissaires ne veulent pas laisser d'équivoque sur le fait que «le secteur privé constitue la pierre angulaire de notre système économique». Ils ajoutent tout de même sans retenue que pour «fonctionner efficacement, il doit être appuyé» (II, 217). Si Donald Macdonald écrit en avant-propos que «les institutions et les politiques gouvernementales peuvent et devraient aider à amortir le choc» (I, xiii) inévitablement produit par le changement auquel les Canadiens sont conviés, les commissaires signalent que l'intervention gouvernementale peut contribuer à aggraver une situation qu'elle «était censée» améliorer (II, 285). Car, invitentils à se rendre compte, «les gouvernements sont incapables de bien prévoir les chocs économiques et de les combattre, afin de prévenir les fluctuations de l'économie» (II, 400). Les gouvernements répondent aux pressions du moment. Pourquoi alors ne pas s'en remettre aux lois du marché?

\section{L'Évangile économique}

Malgré tous les compromis acceptés par les commissaires touchant le respect et la primauté des lois du marché, ces derniers se replient sur des «vérités» essentielles: "les événements ont ébranlé la théorie économique, mais ses principes restent intacts» (I, 145). Pour dire le moins, tel énoncé limite le débat. Si «un courant de pensé [est] très répandu parmi les économistes canadiens", on peut le considérer comme juste. D'autant plus que les «arguments évoqués» reviennent invariablement. C'est ce qui incite les commissaires à conclure «qu'à longue échéance, le Canada profiterait considérablement d'un accord bilatéral de libre-échange avec les États-Unis, notamment deux raisons: la première étant l'accès à un marché plus vaste et dépourvu d'entraves et, la seconde, la 
réalisation d'économies d'échelle» (I, 359). Au fond, qu'importe la constatation faite par les commissaires eux-mêmes que les économistes se sont montrés en désaccord sur d'autres questions comme les niveaux tolérables d'augmentation des prix ou de chômage et que ces désaccords "résultent de différents jugements de valeur et de différentes conclusions sur des questions qui, en principe, sont des questions de fait (II, 310). Décidément, il faut lire Kuttner. Mais les commissaires veulent rassurer: les positions défendues ne sont pas des positions idéologiques. Leur discours sur la privatisation en fait montre.

En dehors de toute idéologie, la question principale se pose comme suit: est-ce que la privatisation [des sociétés publiques] augmenterait leur efficience? Les données disponibles suggèrent que la privatisation de telles sociétés n'occasionnerait probablement pas d'augmentation de l'efficience dans la plupart des cas. Il semble plutôt que, en soi, la privatisation ne produirait guère, ou pas, d'accroissement de productivité (II, 250).

Quiconque autre que les commissaires pourrait se retrouver désemparé. Mais il faut se montrer plus agile. Si la question principale, en debors de toute idéologie, ne conduit pas à la réponse recherchée, il faut se replier sur une autre question et dire que «la privatisation peut augmenter l'efficience du gouvernement lui-même» (II, 252). En dernier recours, il faut rappeler que "privatisation, dé-règlementation et concurrence sont des questions reliées» (II, 250). Or, "les gouvernements restreingnent souvent la concurrence; en fait, ils mènent souvent une lutte intense et permanente contre la concurrence» (II, 241). Ce qui contribue à freiner une nécessaire adaptation économique et à réduire la liberté: «La liberté commence par une reconnaissance de la nécessité» (I, 68). Jugé quasi repréhensible dans l'activité politique (la jungle), le darwinisme peut alors avoir libre cours dans le monde (rationnel) de l'économie.

En l'absence de contraintes, [le processus d'adaptation] se déroule conformément aux lois du marché et s'inscrit dans la poursuite éternelle de la 
maximisation des profits et des revenus. C'est un processus qui implique une sorte de darwinisme économique - la survie du mieux adapté où le marché récompense ou punit l'investissement et les autres décisions (II, 267).

Bien sûr, l'action gouvernementale peut et doit contribuer à amortir les chocs causés par l'adaptation, elle ne doit cependant pas la freiner. Pour les commissaires, l'aide gouvernementale «peut réduire l'opposition politique à l'évolution économique», mais elle peut «également arrêter l'évolution, soit directement ou indirectement, en diminuant les profits liés à l'esprit d'entreprise» (II, 128). Aussi faut-il souligner que «les subventions aux entreprises appartenant aux secteurs en déclin [...] freinent la réorganisation efficace de l'économie et de la création de nouveaux emplois plus durables» (II, 273). De même le gouvernement n'a-t-il pas «de raison de subventionner l'innovation" dans quelque secteur, car «l'innovation est la caractéristique de toute industrie viable» (II, 495). Pour contrer toute réplique faisant ressortir le risque d'un accroissement des problèmes sociaux conséquemment à une réalisation trop brutale de l'adaptation, les commissaires s'appuient sur des données montrant que «le progrès social au Canada dépend [...] manifestement de la vigueur économique du pays» (II, 627). Tout au plus donnera-t-on l'impression de concéder que soient graduellement introduites les réformes au «régime d'assurancechômage du Canada [qui] ne contribue pas réellement à favoriser les adaptations nécessaires au marché du travail, ni à la création d'emplois stables et productifs» (II, 674).

Il demeure pertinent de poser la question du besoin d'une politique industrielle pour favoriser le changement et de demander qui en sera responsable: «Si l'on conclut qu'une politique industrielle conçue en fonction d'objectifs précis est vraiment la meilleure approche du problème, faut-il en déduire que le gouvernement est bien placé pour concevoir et appliquer cette politique?» (II, 145) La réponse vient nette: «Selon nous, le Canada doit opter pour 
une politique industrielle beaucoup plus axée sur le marché qu'elle ne l'est à l'heure actuelle, plutôt que de s'orienter vers une intervention toujours plus grande de l'État»(II, 210). La raison est simple: le marché est meilleur «juge» que les gouvernements.

Choisir les secteurs où nous croyons détenir un avantage par rapport à d'autres pays, c'est prendre pour acquis que les gouvernements sont tout autant, sinon plus, aptes que les marchés, à juger des changements rapides qui interviennent au niveau des demandes des consommateurs et des processus de production. Cette hypothèse se trouve cependant contredite par l'expérience et ne cadre pas avec la souplesse et l'adaptabilité qui nous sont si manifestement nécessaires pour soutenir efficacement la concurrence (I, 55-56).

Les gouvernements ne sont donc pas en mesure d'orienter correctement une politique industrielle favorisant le changement. Et s'il le faut, qu'on les «évite» quand la rationalité économique l'impose. Ainsi, un accord de libre-échange entre le Canada et les États-Unis devrait «soit prendre la forme d'un traité, soit s'appliquer dans le cadre des lois fédérales et des lois des États» du côté américain. Il convient au minimum «d'encourager le gouvernement américain à employer la procédure de voie rapide, lorsqu'il cherchera à obtenir l'approbation pour tout accord relatif au libre-échange». Ainsi, on retrouverait partiellement l'avantage de la démocratie parlementaire qui, au Canada, «assure virtuellement qu'un gouvernement majoritaire réalisera la mise en œuvre législative de tout accord international auquel il aura consenti». Pareilles procédures se justifient «à un moment où le Congrès [américain] est animé par une forte volonté protectionniste». (I, 349)

Comment concilier pareilles positions avec l'importance accordée au gouvernement responsable ou avec la critique qui est faite de l'esprit de parti, ailleurs dans le Rapport? Serait-on traumatisé à ce point par "les excès du nationalisme économique» (I, 63)? Les États ne véhiculent-ils pas de façon abusive les "passions du nationalisme» $(I, 45)$ ? Ce qui risque encore une fois, comme 
le montrent les politiques d'achat des gouvernements (II, 183), d'introduire des distorsions dans les mécanismes «neutres» du marché.

\section{L'abandon d'une politique nationale}

Tout nationalisme serait jugé déphasé si l'on tenait compte que l'on «assiste à une internationalisation de la prise de décision nationale» (III, 47). Les commissaires reconnaissent que «les rôles que jouent les états-nations sur la scène mondiale sont déterminés, pour une bonne part, par ce qui se passe à l'intérieur de leurs frontières» (II, 190). On conçoit cependant que ces mêmes commissaires privilégient l'action progressive du marché contre l'immobilisme des États; les positions antérieurement rapportées le laissent nettement deviner.

À la différence des États, qui sont géographiquement fixes et historiquement enracinés, l'économie de marché est hostile aux frontières nationales, elle crée constamment de nouveaux produits et elle va toujours de l'avant. Sa tendance naturelle est d'organiser et de répartir l'activité économique selon des critères indépendants de l'espace physique, et cette tendance est en contradiction avec l'immobilité des États et leur souci d'assurer la stabilité sociale de leurs citoyens. (I, 46).

Dans le contexte d'une société qui se perçoit comme moderne, il est plus facile de faire adhérer à des positions inspirées du progrès contre d'autres de caractère rétrograde. Surtout quand on suppose que les termes du débat se greffent à une «opposition perfectionniste-protectionniste» $(\mathrm{I}, 315)$. Qui ose vraiment se déclarer ouvertement contre la vertu? Compte tenu du dilemme à résoudre, il est possible que «les gouvernements se trouvent dans une position difficile» (II, 195); le choix s'impose tout de même.

... une chose est claire: si nous voulons, nous Canadiens, résoudre avec succès les graves problèmes auxquels doit faire face notre secteur industriel, le développement économique futur doit s'accorder davantage aux conditions que crée la concurrence internationale qu'aux pressions politiques internes 
pour protéger le statu quo. Devant affronter une concurrence toujours plus forte sur les marchés intérieur et extérieur, il est dans l'intérêt des entreprises des syndicats er de l'État d'augmenter la capacité de l'industrie canadienne d'affronter cette concurrence et de rivaliser avec elle (I, 194).

Tout à fait déterminés, les commissaires n'apparaissent pas à prime abord absolument candides: "L'âge d'or de l'altruisme international, s'il y en a déjà eu un, semble à présent révolu » (I, 208). La confiance (la témérité?) devient dès lors l'attitude disponible. Les ouvertures sont minces un peu partout, tant du côté des États-Unis que du côté du Japon, de l'Europe occidentale, de l'Union soviétique et des pays nouvellement industrialisés.

... l'objectif des commissaires est d'attirer l'attention sur le fait qu'il y a des tendances politiques et économiques intérieures chez nos principaux partenaires commerciaux qu'il faut surveiller, car elles pourraient se révéler des entraves. Dans tous les pays, à l'exception de la Chine qui, de toute façon, ne peut être considérée comme un pays industrialisé, les indices d'une coopération internationale accrue sont, pour le mieux, neutres et parfois très négatifs (I, 209).

Qu'à cela ne tienne, il faut foncer, faire preuve de confiance, affirmer ses convictions. La solution réside dans un accord de libre-échange avec les États-Unis. Le coût sera élevé pour les Canadiens: «L'évaluation des coûts économiques du libre-échange, présentée aux commissaires, indique que le fardeau de l'adaptation sera sans doute plus lourd pour les investisseurs et les salariés canadiens que pour leurs homologues américains» (I, 346). Dans l'esprit des commissaires, il serait cependant illusoire de croire que les Américains ne voient que des avantages à un éventuel accord de libre-échange, même si "le gouvernement américain est généralement favorable au libre-échange». Le Congrès cause problème.

Certains sénateurs et membres du Congrès ont manifesté leur appui au libre-échange entre le Canada et les États-Unis, mais, dans l'ensemble, le Congrès serait très sensible aux vues du milieu d'affaires américain et 
se laisserait fortement influencer par les coûts et avantages sectoriels déterminés par les entreprises individuelles (I, 414).

Qu'importe au fond. Entreprenons d'abord de convaincre les Canadiens et de vaincre leurs réticences. Et si on y est contraint, prévoyons les moyens de brider les Américains.

Un moyen d'assurer une complète suppression du protectionnisme consisterait à rédiger unne disposition stipulant que les mesures de sauvegarde ne pourraient s'appliquer aux producteurs canadiens que si des exportations canadiennes étaient la cause principale de sérieux préjudices subis par des concurrents américains (I, 345).

Quant aux producteurs et commerçants canadiens, il s'agit bien simplement de les amener à penser à long terme, pour qu'ainsi leurs inquiétudes ne masquent pas les effets positifs découlant d'un accord de libre-échange.

Les commissaires [croient] que les incertitudes quant aux effets sont presque toujours partie intégrante du débat politique, même une fois les décisions prises, et qu'on ne peut présumer que le maintien du statu quo soit toujours le meilleur mode de fonctionnement. Nous sommes convaincus que sur ce sujet important, le poids de la preuve va dans la direction que nous avons décrite. Par conséquent, nous sommes convaincus qu'il faut changer la situation, car il est important d'améliorer et de garantir l'accès canadien aux marchés d'exportation; nous sommes néanmoins disposés à continuer de discuter ces questions dans le débat qui suivra sans aucun doute $(I, 366)$.

Il faut somme toute lutter contre le courant, manifeste à travers «le degré inquiétant de protectionnisme qui refait surface dans les échanges internationaux», spécialement par «un recours accru aux barrières non tarifaires» (I, 178). Cette situation, due aux «interventions croissantes de l'État», vient contrer et même annuler «les réductions tarifaires mises en ouvre dans le cadre des accords du GATT» (I, 144). Une première preuve de bonne volonté pourrait s'appuyer sur la remise en question des efforts de "canadianisation», forme d'intervention dont la valeur «est très douteuse. Une réduction du nombre de ce type d'interventions 
constitue une première étape, fondamentale, pour contrôler la croissance du gouvernement et du secteur mixte». (II, 254) Il convient alors de gommer «l'absence d'innovation attribuable au fait que notre économie est dominée par des filiales d'entreprises étrangères» (I, 300). Il est vrai que les commissaires se disent forcés «de constater que la relation entre la productivité et la capacité de faire concurrence sur les marchés internationaux n'est pas aussi directe que cela peut sembler à prime abord» (I, 166). La solution, a-t-on encore besoin de le répéter, ce sont les marchés eux-mêmes. Le reste viendra par surcroît...

À ceux enfin qui osent se demander si «notre autonomie et notre identité ne seront pas compromises par le libre-échange», les commissaires font valoir «qu'une intégration commerciale accrue [ne] menace [pas] la survie ou l'épanouissement de l'identité canadienne, car l'interdépendance commerciale, l'autonomie politique et l'identité culturelle ne sont pas incompatibles».

La nation canadienne n'est dès lors, nullement condamnée à disparaître en raison de ses relations commerciales plus libres et plus sûres avec son voisin du sud. Le libre-échange réduira nos divisions régionales et nous inspirera une confiance accrue. Le sentiment national canadien et notre identité collective comme peuple sont enracinés dans plus d'un siècle d'histoire commune. Il n'est pas irréaliste d'affirmer que notre sentiment d'appartenance au Canada se verra renforcé par la juxtaposition d'échanges commerciaux plus intégrés à un système gouvernemental canadien autonome (I, 67).

L'importance accordée au sentiment d'appartenance semble pratiquement supplanter les réticences des commissaires à reconnaître la nécessité d'exceptions à un accord global de libre-échange. Aussi sont-ils prêts à admettre, qu'en plus des secteurs de l'énergie et d'autres ressources naturelles et du cas d'industries de services particulières, "les activités culturelles [puissent] faire également l'objet d'un traitement spécial» (I, 338). Néanmoins, une fois assurée la pérennité de l'identité collective canadienne, la lucidité 
demeure toujours de mise. Dans le cadre d'un aveu dont ils s'empressent de réduire la portée, les commissaires ne parviennent pas à éluder complètement le scénario d'une perte relative d'autonomie politique.

... dans un contexte propice au libre-échange ou aux échanges commerciaux plus ouverts avec les États-Unis, il serait encore plus important que certaines de nos politiques gouvernementales soient plus ou moins semblables à celles des États-Unis pour que nos industries puissent être raisonnablement concurrentielles. Ceci ne signifie pas que tous les aspets de notre politique industrielle devraient être nécessairement identiques à l'approche préconisée par les États-Unis (II, 221).

Si l'annexion politique est rejetée, le compromis demeure de taille. D'ailleurs, les commissaires font déjà remarquer que «les marchés internationaux de capitaux se sont tellement développés que les banques centrales se plaignent souvent que les ressources dont elles disposent ne leur permettent pas de résister aux pressions de ces marchés» (II, 360). À leur avis, la voie à suivre n'est pas tracée du côté d'une intervention sur la liberté de mouvement des capitaux, même si cette liberté entraîne que «nos taux d'intérêt se trouvent essentiellement hors de notre contrôle» (II, 362). Il s'agit alors d'accepter un état de faiit.

L'économie mondiale est maintenant beaucoup plus intégrée qu'elle ne l'était il y a vingt ou trente ans. En particulier, le marché mondial des capitaux s'est radicalement transformé, dans son ampleur aussi bien que dans son efficacité, et cela diminue l'autonomie dont dispose le gouvernement d'une économie ouverte en matière de politiques macro-économiques (II, 324).

Pour tout dire, l'autonomie politique est devenue une question de degré, qu'on aime cela ou pas. Ce qui compte, ce sont les bénéfices économiques que l'on compte retirer d'une acceptation plus poussée de la concurrence internationale. À la limite, on pourrait songer à exiger des directeurs canadiens d'entreprises qu'ils donnent «aux intérêts canadiens une considération sérieuse 
et continue, lorsqu'ils prennent des décisions importantes qui concernent leur société» (II, 266). Pourquoi alors ne pas s'inspirer du fonctionnement de l'Agence d'examen de l'investissement étranger qui «a encouragé un dialogue pragmatique entre les fonctionnaires du gouvernement et les investisseurs étrangers et a clairement défini d'avance les normes canadiennes de bonne conduite dans le monde des affaires»? Même si "les commissaires pensent $[\ldots]$ que les débats publics récents au sujet de [l'Agence] ont eu tendance à exagérer ses effets coercitifs ou restrictifs» (II, 263), les développements récents qui ont conduit à son démantèlement obligent à noter que la liberté des investisseurs et administrateurs d'entreprises tolère mal le compromis. Un accord de libre-échange créerait sans doute un monde tout à fait nouveau.

\section{Un fédéralisme fonctionnel}

Un des volets importants du mandat de la Commission Macdonald touchait l'état de l'union économique canadienne et les problèmes que cela soulevait dans le rapport de l'économie canadienne à l'économie mondiale. Les commissaires ne l'oublièrent pas: "la création de cette Commission est, en grande partie, le résultat de l'inquiétude suscitée par les politiques des deux niveaux de gouvernement qui érodaient [...] l'union économique et réduisaient le rendement de l'économie nationale» (III, 110). Dans cette partie de leur rapport, les commissaires semblent cependant prêts à prendre leur distance vis-à-vis un certain économisme: "L'édification d'une union économique canadienne n'a de sens que parce que nous sommes avant tout une communauté politique nationale» (idem). Tout en pensant que "notre performance économique dépend des bonnes relations entre les gouvernements fédéral et provinciaux», les commissaires refusent de trancher en termes absolutistes. 
En gardant cette réalité à l'esprit, les commissaires préconisent une approche plus fonctionnelle du fédéralisme. Au lieu de nous demander simplement s'il faut centraliser ou décentraliser, ou accroître le pouvoir de l'État fédéral ou des provinces, nous devrons chercher à nous préoccuper réellement des besoins des citoyens et à administrer nos politiques économiques et sociales avec efficacité. Nous recommandons par conséquent que l'on fasse preuve d'une plus grande souplesse dans nos réponses aux problèmes que pose l'interdépendance gouvernementale (III, 11).

Voulant être bien compris, les commissaires affirment ne pas vouloir sanctionner «les moyens inconstitutionnels pour obtenir des fins nationales». Ils soulignent cependant que «vouloir nier la contribution de la direction et de l'initiative fédérales revient à nier l'histoire des cinquante dernières années» (III, 30). Contrairement à ce que l'on retrouvait dans le Rapport Pépin-Robarts, les commissaires s'inspirent plutôt du Rapport Rowell-Sirois pour établir que «peu de raisons incitent à croire que la seule centralisation permettrait une planification cohérente» (III, 165). À la différence des thèses défendues il y a quelques années par les gouvernements fédéral et ontarien, ils s'entendent plutôt pour dire que «malgré les manifestations récentes d'inquiétude sur la balkanisation et la fragmentation progressives de l'économie, l'union canadienne fonctionne néanmoins, de façon efficace en général» (III, 150). Bien sûr, les limites interprovinciales au commerce existent, mais il importe de ne pas dramatiser, car les distorsions ainsi introduites ont finalement "peu d'effet sur les coûts de production" (III, 132).

Dans une certaine mesure fidèles au modèle d'analyse économique qu'ils privilégient, les commissaires apparaissent au moins partiellement influencés par le cadre théorique élaboré par Albert Breton (III, 554-600). De cette manière, ils «ne s'opposent pas à la coopération, mais bien à l'argument voulant qu'elle soit la valeur suprême par excellence». Ils vont encore plus loin: «La coopération entre les deux ordres de gouvernement ne peut constituer l'essence du fédéralisme ni servir de critère déterminant pour en évaluer le résultat. Poussée à l'extrême, l'insistance sur la coopération 
détruit le fédéralisme». En dernière analyse, s'opposer à la concurrence entre les gouvernements «équivaudrait à préconiser implicitement un État unitaire, ce qui n'est ni possible, ni souhaitable pour le Canada» (I, 74).

La concurrence entre gouvernements, le chevauchement des tâches et le double emploi dans les services sont inhérents au fédéralisme. En réalité, ils comptent parmi ses principaux avantages. Une décision politique est susceptible de répondre davantage aux aspirations des citoyens, lorsque ceux-ci peuvent s'adresser à un autre ordre d'autorité si le premier ne tient pas compte de leurs préoccupations, surtout lorsque ces deux ordres de gouvernement rivalisent pour se mériter la confiance des électeurs (III, 287).

Au total, les commissaires estiment que «dans la plupart des cas l'autorité que la Constitution confere au gouvernement fédéral est suffisante» et que, par ailleurs, «les pouvoirs provinciaux ne réduisent pas la capacité du gouvernement fédéral de résoudre les problèmes». Ce qui les amène à établir: «nous ne trouvons aucun motif qui puisse nous inciter à accroître sensiblement la centralisation ou à nous faire l'apôtre de la décentralisation" (III, 293). Les commissaires se sentent donc justifiés de s'abstenir de recommander une modification du "partage des compétences" (III, 291), même s'ils admettent par ailleurs que «les divisions constitutionnelles [peuvent] compliquer davantage la situation" quand il s'agit de définir des politiques industrielles (II, 154). Si des réformes s'imposent, elles doivent partir de ce qui est.

Les réformateurs ne peuvent pas tout effacer et tout recommencer. Ils doivent partir du réseau de relations institutionnelles, du pouvoir déjà en place et de l'interdépendance entre l'État et la société que les politiques passées ont établi [sic] (III, 34).

Une des recommandations essentielles des commissaires favorise la mise au point d'un «code de conduite économique» non incorporé à la Constitution, qui permettrait aux gouvernements du Canada de «préciser les politiques et les pratiques acceptables 
ou non dans le cadre de l'union économique canadienne» (III, 152). D'un point de vue plus formel, les commissaires sont tout de même «d'avis qu'il faudrait renforcer la protection constitutionnelle de l'union économique [...] contre les barrières tarifaires [pour] englober tout autant les services que les biens" dans l'application de l'article 121 de la Loi constitutionnelle de 1867 (III, 151). Se démarquant des positions véhiculées par le fédéral il y a quelques années, les commissaires parlent de la prépondérance des interventions du fédéral "avec précaution»; ils préferent visiblement une approche fonctionnelle: "Tout comme l'activité fédérale dans le domaine de la gestion de l'économie n'interdit pas l'implication provinciale, il y a, et il doit y avoir, une conscience des intérêts nationaux de la part des provinces qui assument ces responsabilités» (III, 167).

Partir de ce qui est en place, c'est aussi tenir de la Charte des droits et libertés adoptée en 1982 qui, par son article 6, affirme le droit des Canadiens à la mobilité. Dès lors, la Commission se montre «convaincue que le gouvernement du Canada a un rôle important à jouer dans l'enseignement postsecondaire et la formation professionnelle» (II, 818). Pour traduire de façons additionnelles une approche fonctionnelle du fédéralisme, d'autres propositions des commissaires pourraient être citées allant d'une coordination plus poussée des budgets fédéral et provinciaux (III, 179) à la préservation de la responsabilité fédérale dans le «fonctionnement du programme de péréquation" (III, 219). En dernière analyse, il importe aux commissaires que la population perçoive le gouvernement comme représentatif des intérêts pour qu'il soit mieux «en mesure de promouvoir des compromis, d'assurer un leadership dynamique et d'instaurer le consensus nécessaire à l'acceptation volontaire de ses décisions par les citoyens» (III, 77). Compte tenu des effets attendus du programme économique proposé par le Rapport Macdonald, ce dernier énoncé n'est pas sans signification. 
C'est en grande partie le fondement de la proposition des commissaires de rendre le Sénat électif par mode de représentation proportionnelle (III, 97-98). En somme, les commissaires sont amenés à penser que le système fédéral ne peut pas être conçu «uniquement en fonction du marché et [que] cela est essentiel à la négociation au sein de la Confédération» (III, 145). Dans une certaine mesure, on s'arrête à la question de l'union économique canadienne faute de l'édification d'un véritable marché multinational (III, 125).

D'ici l'avènement de ce monde annoncé et souhaité par les commissaires, il convient d'éduquer les Canadiens pour les y préparer, pour amortir le choc. Quand le moment sera venu, le Canada aura-t-il droit à un statut confirmant son "caractère distinct", comme on le recommande pour le Québec dans le cadre du préambule de la Constitution (III, 383)? Reconnaîtra-t-on à ce pays un droit de veto semblable à celui recommandé pour le Québec quand sa spécificité ou la dualité canadienne sônt remises en cause (III, 388)?

Semblables questions seront sans doute qualifiées de tendancieuses; elles risquent tout au moins d'être perçues comme irrationnelles. Car les commissaires ont bien présenté le changement comme inéluctable. Il ne reste qu'à se rallier. Accepter et faire face au changement n'est pas nécessairement synonyme de démission politique, même si la politique n'est pas aussi froidement rationnelle que l'économique.

\section{Conclusion: Leçons et histoire}

La lecture de l'histoire risque de montrer la fragilité de l'échafaudage théorique de la Commission Macdonald. À vouloir trop s'en remettre aux experts, les commissaires ont peut-être perdu de vue une bonne partie du Canada «réel». D'une section 
à l'autre, ci-haut, on perçoit bien que l'inspiration ne fut pas monolithique. La procédure de rédaction suivie pouvait difficilement produire d'autre résultat.

Les premières ébauches furent rédigées par huit équipes coordonnées par des spécialistes, parmi lesquels figuraient des chercheurs séniors en sciences économiques, en science politique et en droit, ainsi que des analystes du secrétariat, qui condensèrent et résumèrent la documentation recueillie au cours des audiences. Individuellement et en groupe, pendant presque un an d'intenses discussions, les commissaires révisèrent, réaménagèrent, supprimèrent et intégrèrent différentes ébauches (I, xxi).

L'intégration est mal réussie. La transition d'une salle de cours à l'autre est mal assurée. En s'acharnant à vendre leur salade respective à base de principes, les experts de la Commission ne parviennent finalement qu'à susciter un certin scepticisme quant aux préceptes qu'ils en font découler. Quand on enseigne sur la politique, on ne la fait habituellement pas, par crainte de la frustration. Car les principes et les «lois» (scientifiques) demeurent «théoriques» et ne peuvent être qu'adaptés dans l'action. C'est alors qu'à vouloir trop éduquer, on arrive peut-être à négliger l'écoute et l'observation. On risque à tout le moins de n'être attentif qu'aux premiers de classe; eux, ils comprennent. La teneur de quelques commentaires publiés dans le volume III du Rapport tend à valider l'analogie.

Le traitement fait pas Judith Schlanger de l'évolution discontinue du développement intellectuel suggère une autre analogie intéressante.

Si la thématique "lamarckienne» est celle de l'accomodation fonctionnelle, la thématique "darwinienne» est évidemment celle de la variation ou mutation, et celle de la sélection par concurrence. Plus encore, le propre de cette thématique est de maintenir les deux aspects séparés. La mutation génétique est dissociée des procédures de la sélection; c'est ce qui rend possible un récit non téléologique du parcours. C'est ce qui permet de 
ne pas finaliser l'histoire. Mais lorsqu'il s'agit des idées, peut-on vraiment dire que l'engendrement des théories n'est pas orienté, n'est pas sélectif ${ }^{14}$ ?

Comment ne pas faire le rapprochement avec le contenu du Rapport Macdonald qui balance entre son darwinisme économique et son approche politique fonctionnelle? Cela oblige le lecteur à des sauts qualitatifs importants, au point qu'il n'est pas toujours certain de bien suivre. Les nombreuses données rassemblées par l'imposant appareil de recherche de la Commission auraient pu parfois falsifier certains principes théoriques. Mais, le Rapport le dit bien, parce que partagés et répétés, les principes tiennent. Sans doute s'est-on inspiré de Karl Popper pour se convaincre que le tout demeure scientifique et rationnel, parce que l'univers des réfutations possiblees n'est pas complètement épuisé.

Malgré qu'ait longtemps duré la conviction que le soleil tournait autour de la terre, la terre n'en continuait pas moins de tourner. Vouloir faire partager sa conviction que le monde réel se retrouve condensé dans le marché n'empêchera pas les Canadiens de faire de la politique, même si on veut leur faire croire que c'est irrationnel. Et c'est en grande partie comme cela que le Canada de l'an 2000 a quelque chance de se construire.

À moins que les principes dogmatiques, enrobés d'expertise, parviennent à totalement convertir. Dans un tel cas, le Rapport Macdonald aura quand même réussi l'exploit de faire voir un Sinclair Stevens plein de lucidité quand il déclarait en mars 1985: «Au Canada, nous lisons les manuels d'économie et les appliquons, alors que les Américains ne négocient rien sans avoir consulté leur propre industrie» ${ }^{15}$

14. Judith Schlanger, L'invention intellectuelle, Librairie Arthème Fayard, Paris, 1983, pp. 150-151.

15. Cité par Miville Tremblay, "Stevens envisage la possibilité d'assurer une partie du marché canadien à certaines industries", dans Le Devoir, 25 mars 1985, p. 11. 